\title{
Effects of Regular and Irregular Deep Brain Stimulation on the Basal Ganglia Dynamics: A Computational Approach
}

\author{
Seyed Mojtaba Alavi ${ }^{1}$, Amin Mirzaei ${ }^{2}$, Reza Ebrahimpour ${ }^{1 *}$ \\ ${ }^{1}$ Faculty of Computer Engineering, Shahid Rajaee Teacher Training University, Tehran, Iran \\ ${ }^{2}$ Institute of Neuroscience and Medicine (INM-6), Juelich, Germany
}

\section{A BSTRACT}

Introduction: The basal ganglia are subcortical structures of the brain which are involved in the motor system. One of the common disorders related to malfunctioning of the basal ganglia is Parkinson's disease (PD). PD is accompanied by strong and permanent beta band oscillations in the basal ganglia. Deep brain stimulation (DBS) of the subthalamic nucleus (STN) in the basal ganglia is known as an approach to reduce pathological beta oscillations in PD and to improve PD-related motor symptoms. Despite the success of this approach, its underlying mechanisms are still unclear. Materials and Methods: In this study, we proposed a computational network model for the cortico-basal ganglia loop based on the model suggested by Terman and colleagues earlier. We modified the network model in the manner that it can generate pathological beta oscillations in the PD state. Then, by applying different DBS scenarios (such as regular and irregular high frequency excitation of STN), we investigated which scenario is able to reduce the pathological beta power more efficiently. Results: Our results show that the network model can generate pathological beta band oscillations similar to what has experimentally been observed in the PD state. Our simulation results indicate that the regular DBS outperforms the irregular DBS, in terms of reducing the PD-related beta oscillations and improving the motor symptoms. Conclusion: Using our simulation results, we conclude that applying regular high frequency DBS on the STN neurons in the basal ganglia can ameliorate PD-related motor deficits better than irregular DBS pulses. This simulation result is experimentally testable.

\section{Key words:}

1. Basal Ganglia

2. Subthalamic Nucleus 3. Deep Brain Stimulation

*Corresponding Author: Reza Ebrahimpour

E-mail: rebrahimpour@sru.ac.ir 


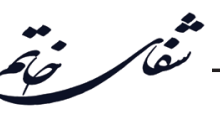

\title{
اثرات تحريك عميق مغزى منظم و نامنظم روى ديناميك عقدههاى قاعدهاى: يك رويكرد محاسباتى
}

\author{
سيد مجتبى علوى'، امين ميرزايى '، رضا ابر اهيميور'". \\ 'دانشكده مهندسى كامييوتر، دانشخاه تربيت دبير شهيد رجايى، تهران، ايران \\ 'بيروهشكدة علوم اعصاب و دارويى، يوليش، آلمان
}

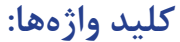

1 إ. عقدهاى قاعدهاى

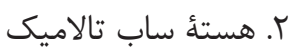

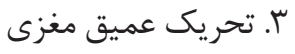

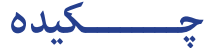

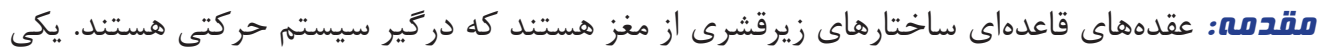

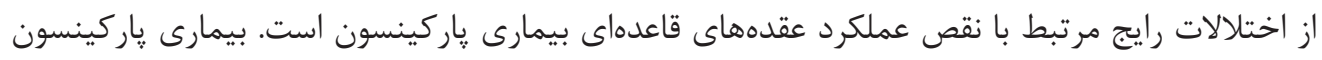

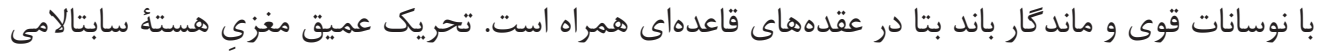

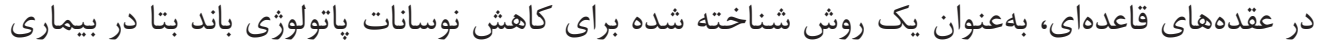

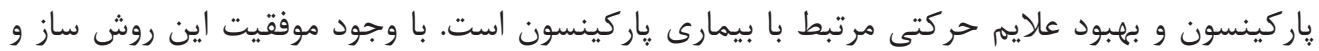

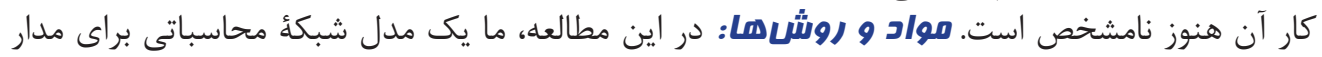

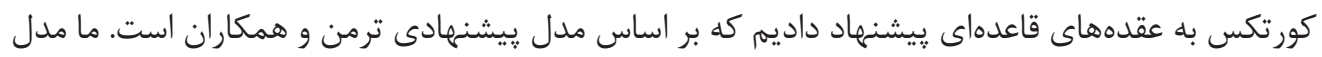

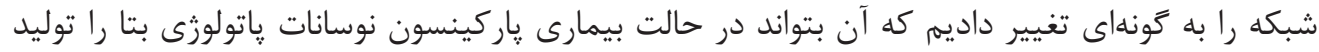

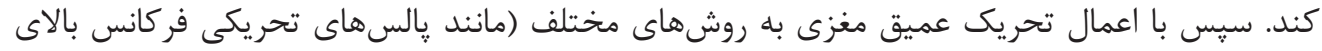

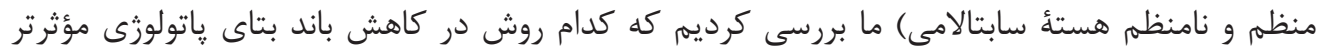

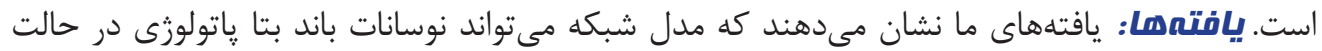

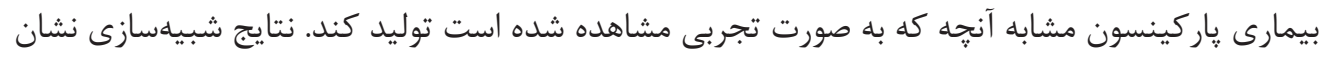

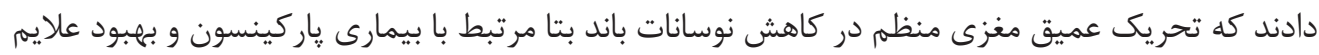

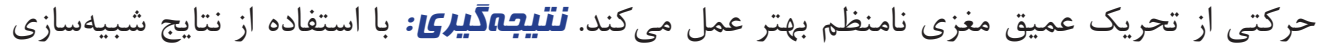

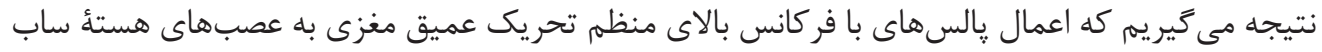

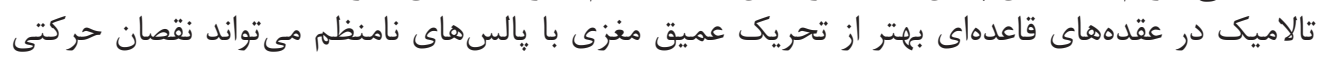

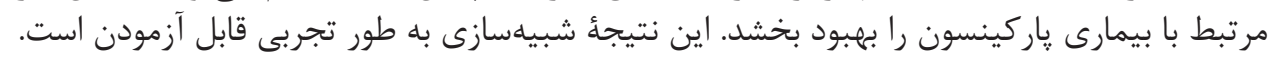




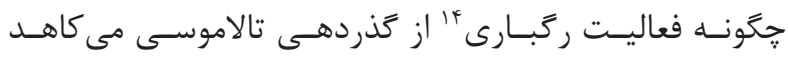

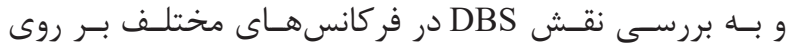

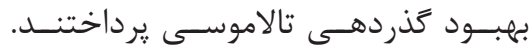

يكىى از روشهـاى مطالعـات در علوم اعصاب، مدلسـازى اسـت.

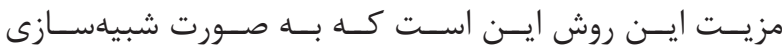

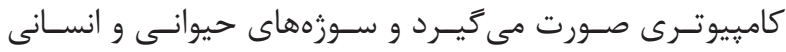

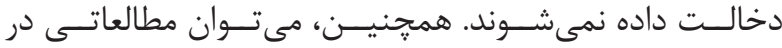

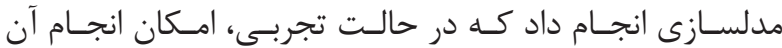

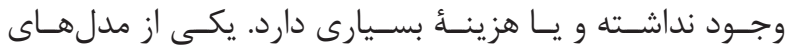

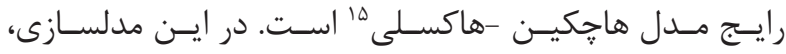

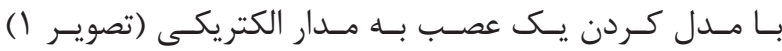

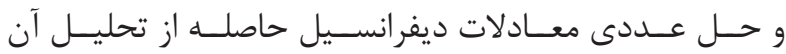

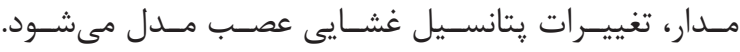

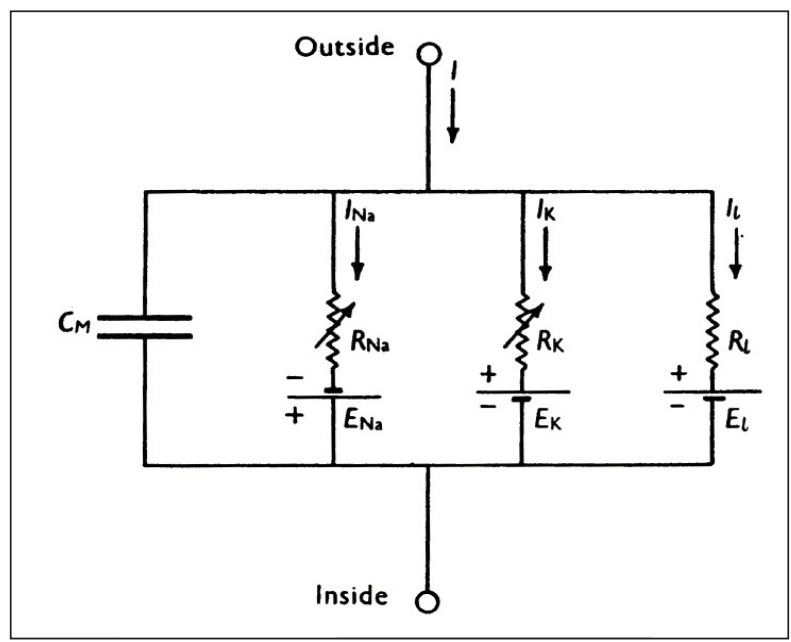

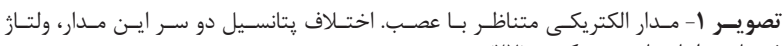

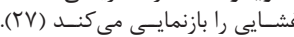

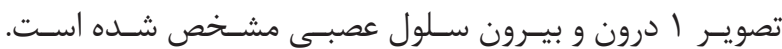

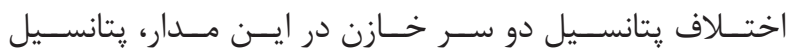

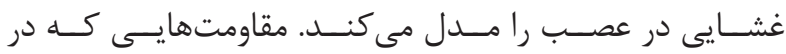

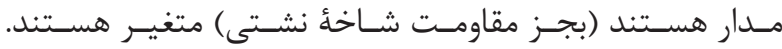

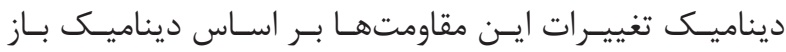

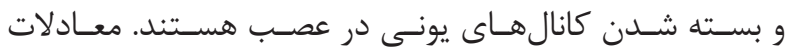

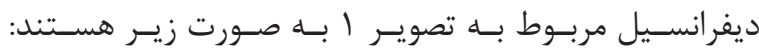

$C_{M} V^{\prime}=-\bar{g}_{l}\left(V-E_{l}\right)-\bar{g}_{N a} m^{\psi} h\left(V-E_{N a}\right)-\bar{g}_{K} n^{\varphi}\left(V-E_{K}\right)$

$$
\begin{aligned}
n^{\prime} & =\alpha_{n}(V)(1-n)-\beta_{n}(V) n \\
m^{\prime} & =\alpha_{m}(V)(1-m)-\beta_{m}(V) m \\
h^{\prime} & =\alpha_{h}(V)(1-h)-\beta_{h}(V) h
\end{aligned}
$$

\footnotetext{
${ }^{1}$ Basal ganglia

${ }^{2}$ Subcortical

${ }^{3}$ Sub thalamic nucleus

${ }^{4}$ Globus pallidus

${ }^{5}$ Striatum

${ }^{6}$ Substantia nigra pars compacta and pars reticulata

${ }^{7}$ Inhibition

${ }^{8}$ Synaptic connections
}

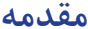

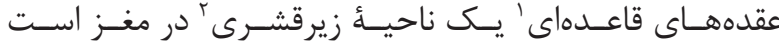

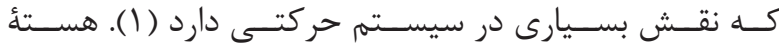

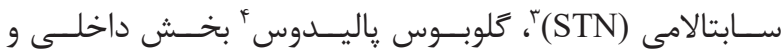

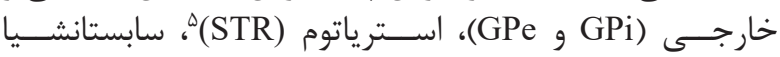

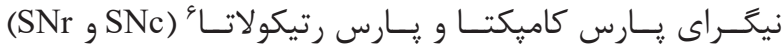

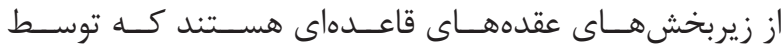

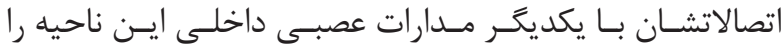

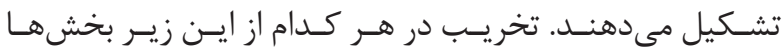

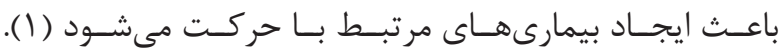

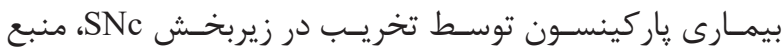

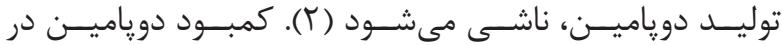

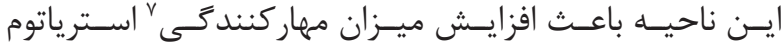

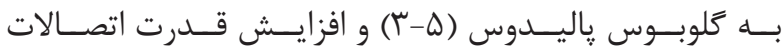

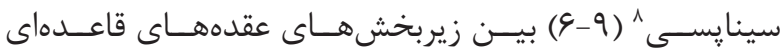

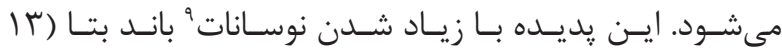

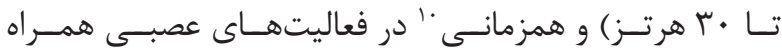

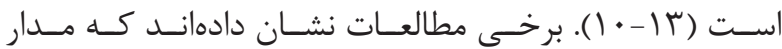

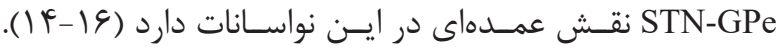

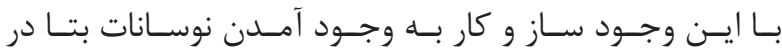

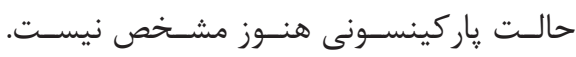

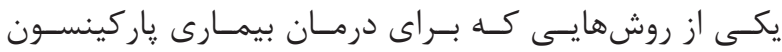

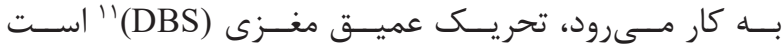

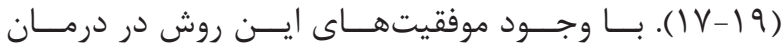

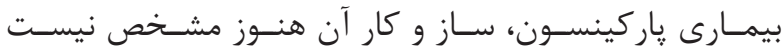

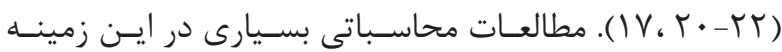

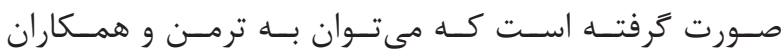

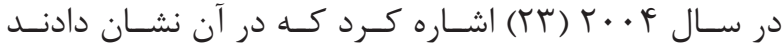

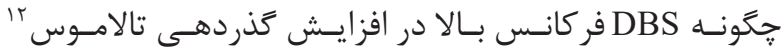

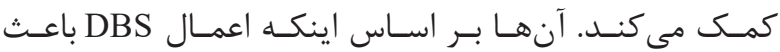

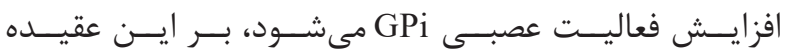

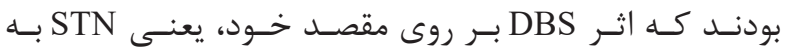

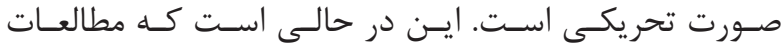

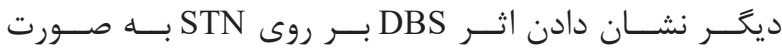

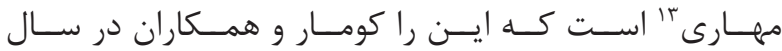

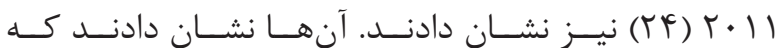

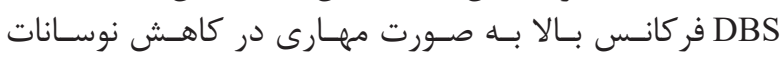

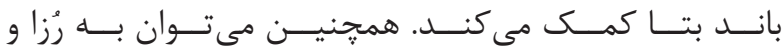

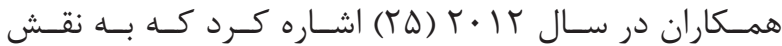

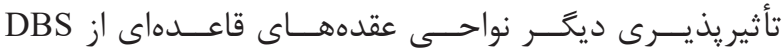

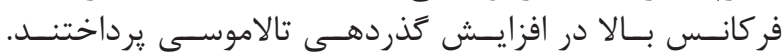

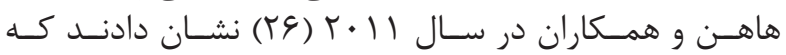

\footnotetext{
${ }^{9}$ Oscillations

${ }^{10}$ Synchrony

${ }^{11}$ Deep brain stimulation

${ }^{12}$ Thalamus fidelity

${ }^{13}$ Inhibitory

${ }^{14}$ Bursting activity

${ }^{15}$ Hodgkin-Huxley
} 


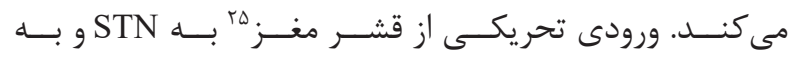

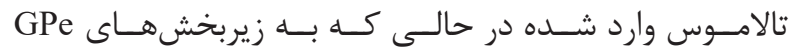

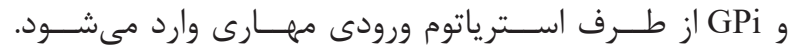

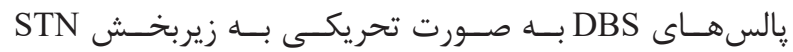

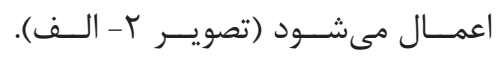

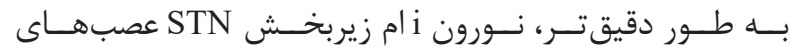

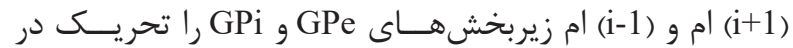

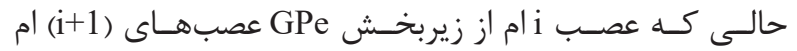

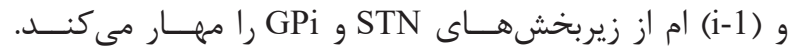

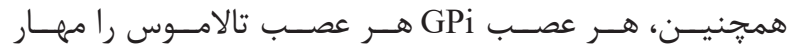

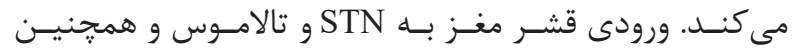

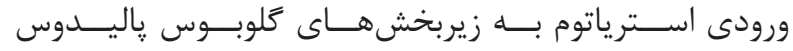

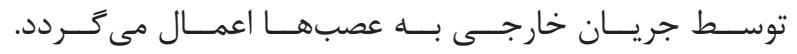

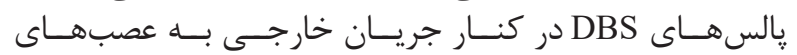

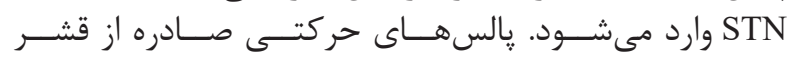

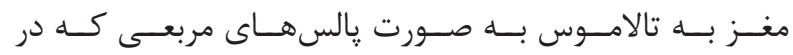

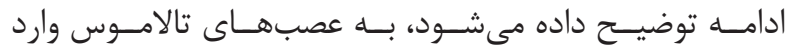

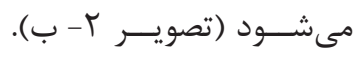

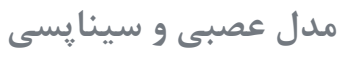

مــدل عصبـى عصبهــاى اســفاده شــده در ايـن مطالعــه

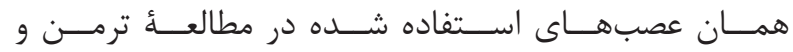

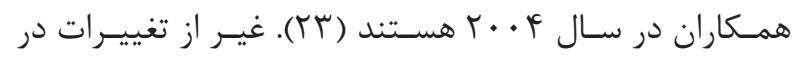

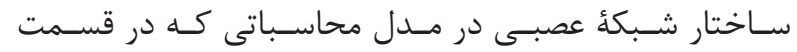

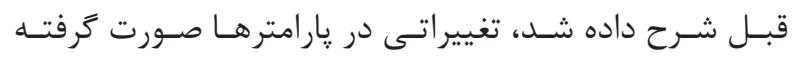

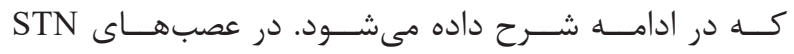

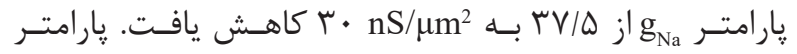

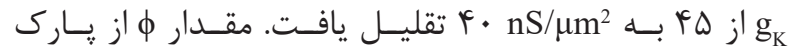

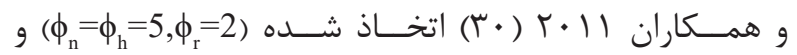

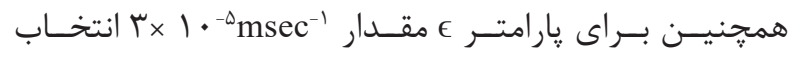
شـده اســت يارامتــر سـلامت nS/

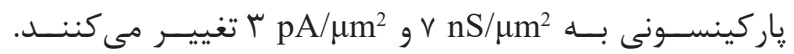

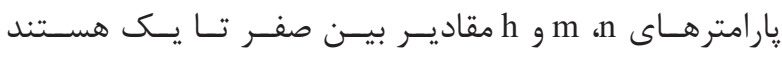

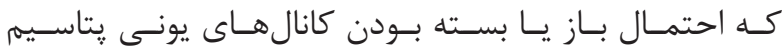

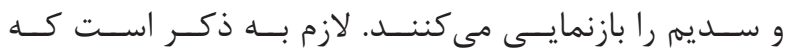

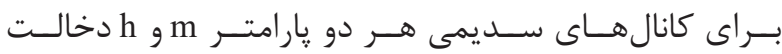

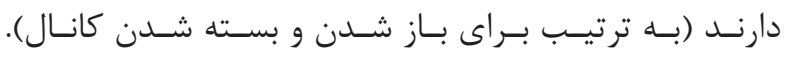

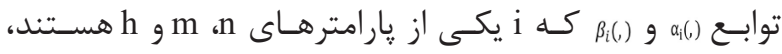

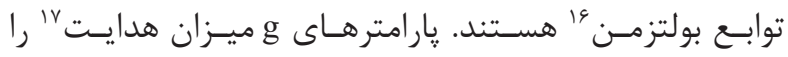

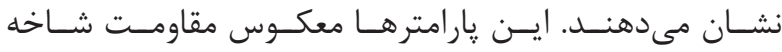

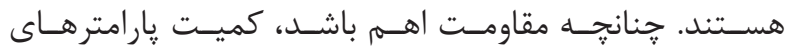

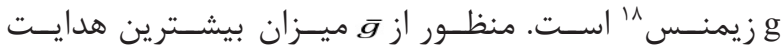

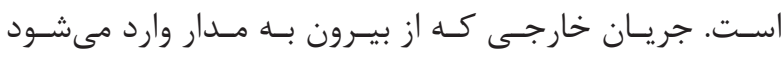

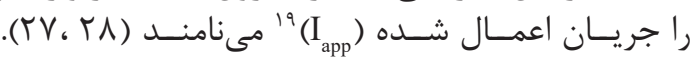

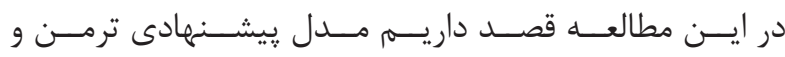

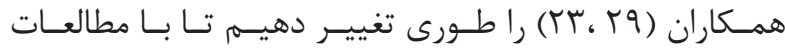

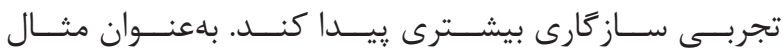

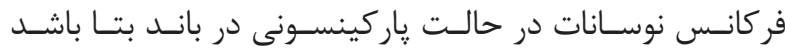

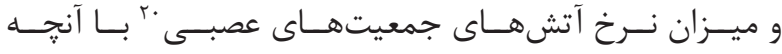

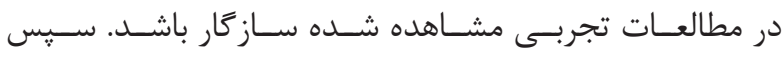

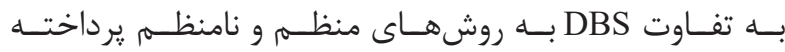

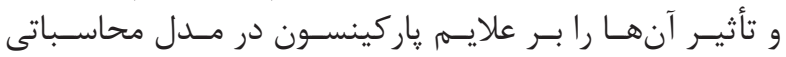

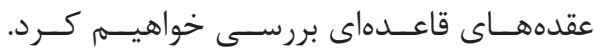

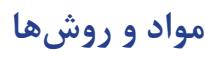
ساختار مدل شبكه

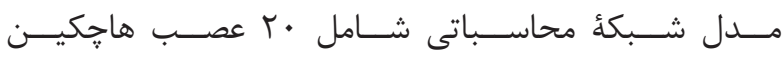

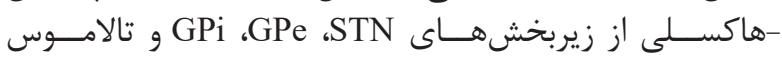

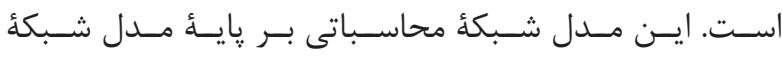

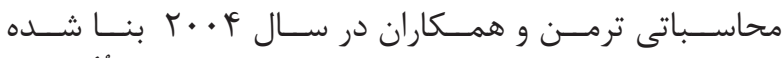

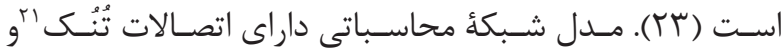

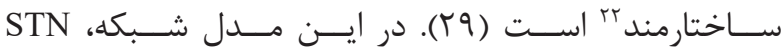

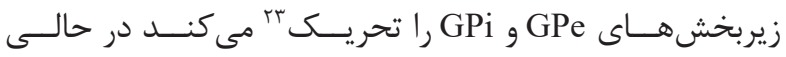

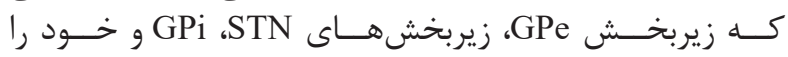

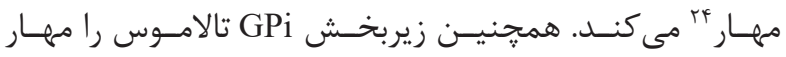

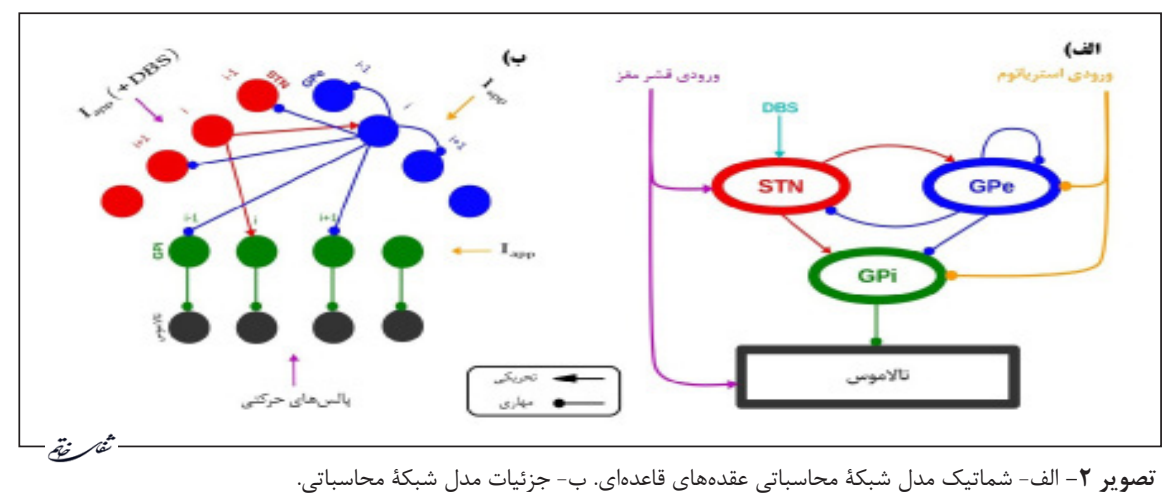

\footnotetext{
${ }^{16}$ Boltzmann

${ }^{17}$ Conductance

${ }^{18}$ Siemens

${ }^{19}$ Applied current

${ }^{20}$ Population firing rate
}

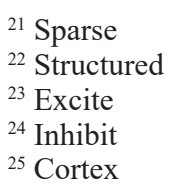




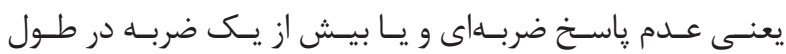

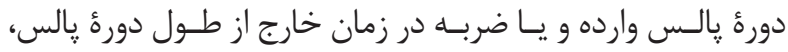

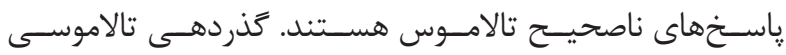

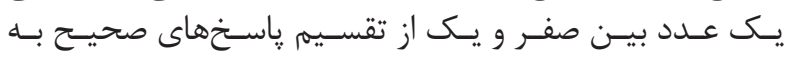

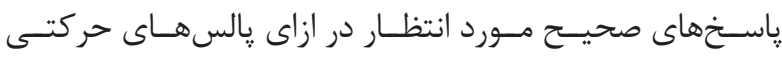

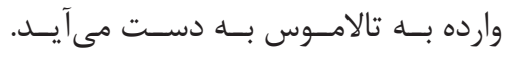
زمان -فعاليت جمعيت عصبى

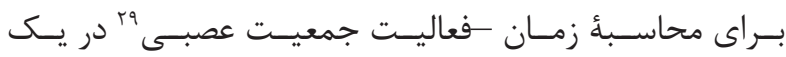

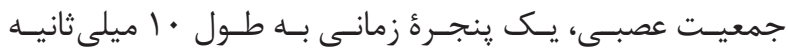

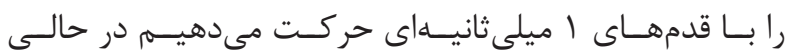

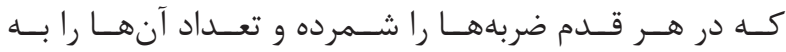

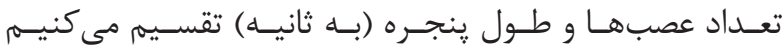

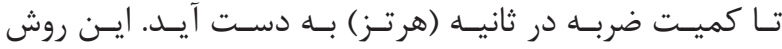

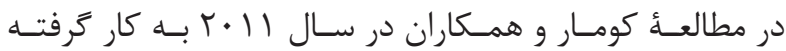

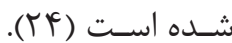

شاخص همزمانى

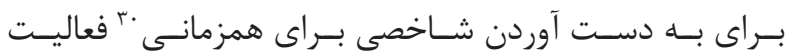

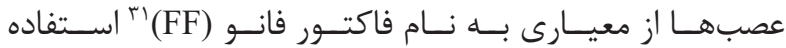

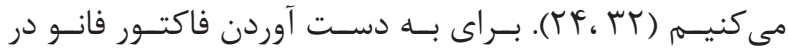

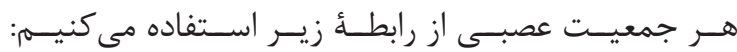

$$
\mathrm{FF}=\frac{\operatorname{Var}(P F R)}{E(P F R)}
$$

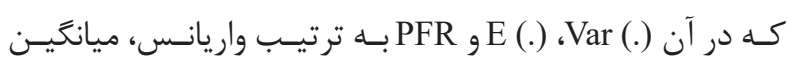

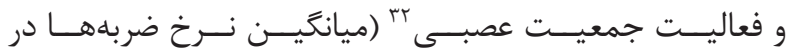

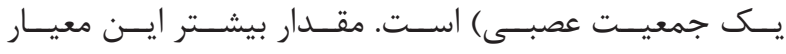

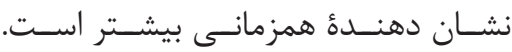
ميانغين شدت توان طيف فر كانسى

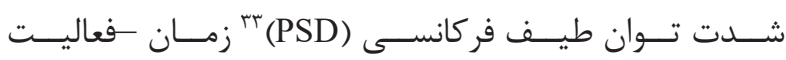

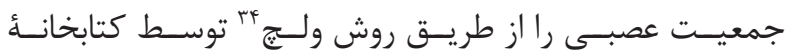

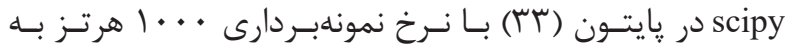

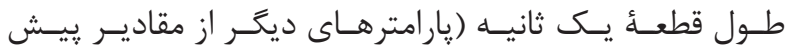

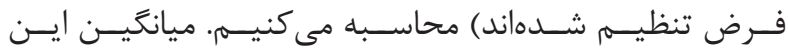

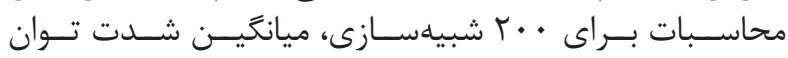

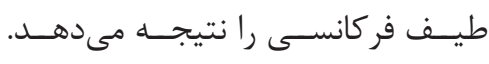
شاخص نوسان

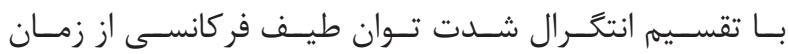

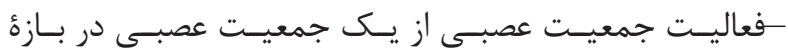

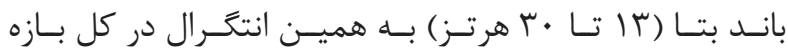

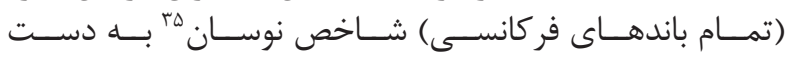

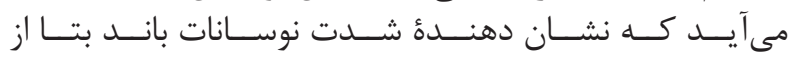

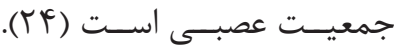

${ }^{26}$ Conductance based

${ }^{27}$ Pulse duration

${ }^{28}$ Spike

${ }^{29}$ Time resolved population ring rate

${ }^{30}$ Synchrony index

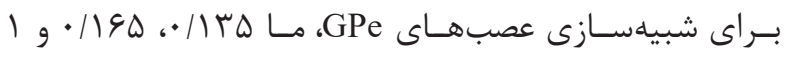

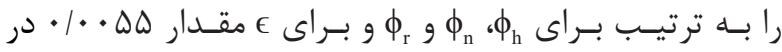

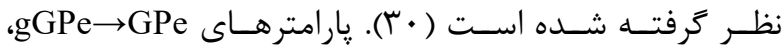

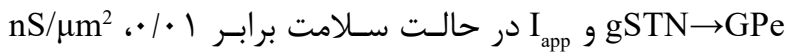

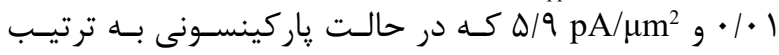

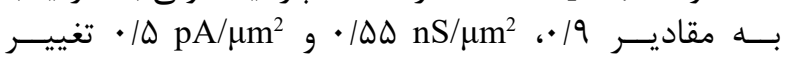

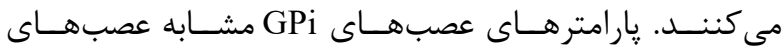

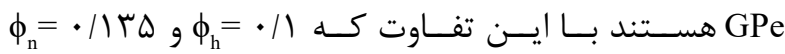

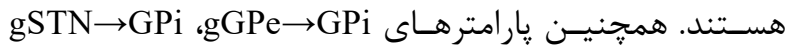

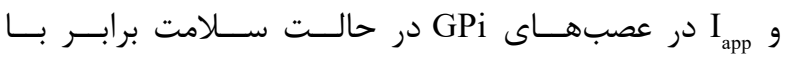

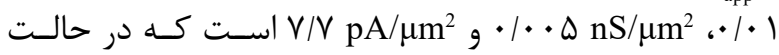

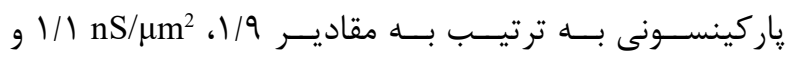

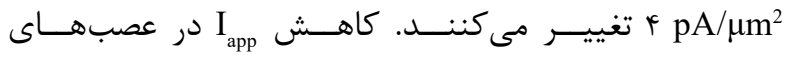

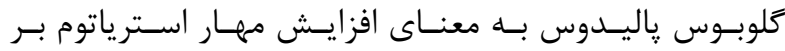

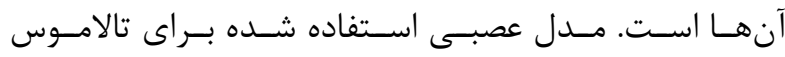

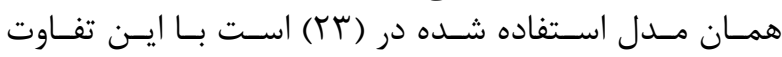

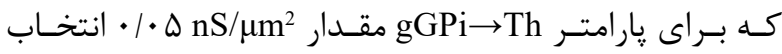

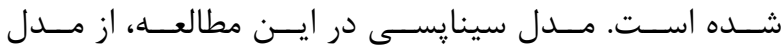

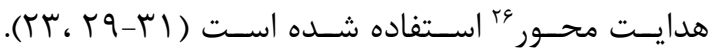

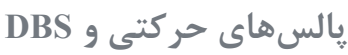

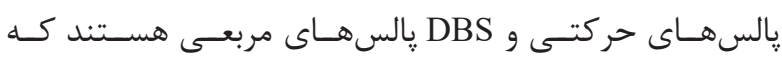

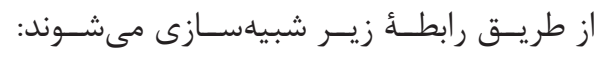

$\mathrm{I}_{\text {pulse }}=A \times H\left(\sin \left(\frac{2 \pi f t}{1 \cdots}-r n d\right)\right) \times\left(1-H\left(\sin \left(\frac{2 \pi f(t+\delta)}{1 \cdots}-r n d\right)\right)\right)$

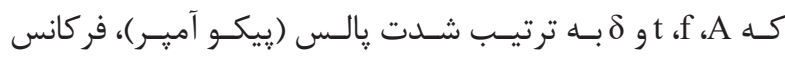

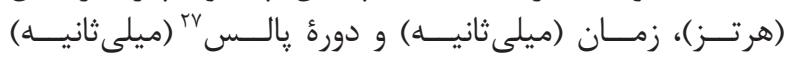

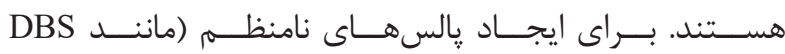

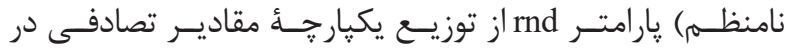

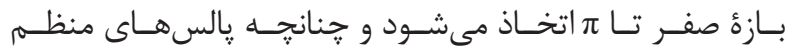

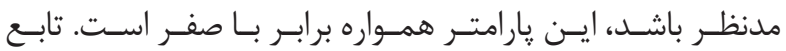

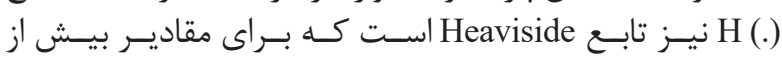

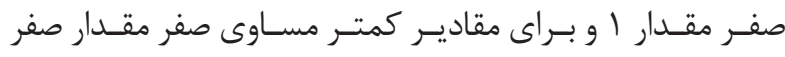

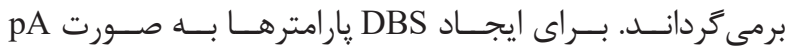

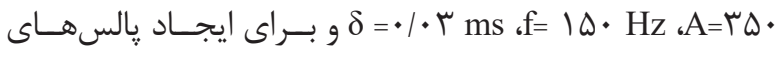

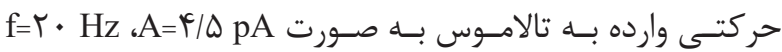
و

$$
\text { كذردهى تالاموسى }
$$

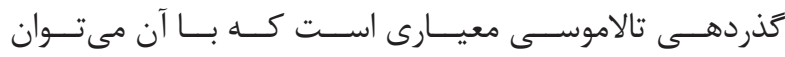

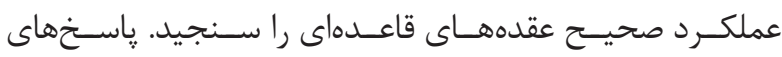

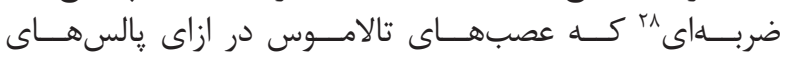

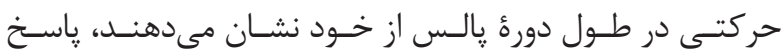

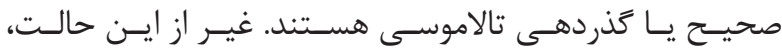

${ }^{31}$ Fano factor

${ }^{32}$ Population firing rate

${ }^{33}$ Power spectral density

${ }^{34}$ Welch's method

${ }^{35}$ Oscillation index 


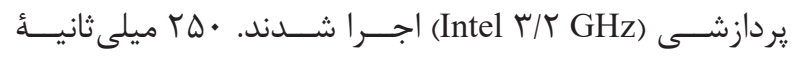

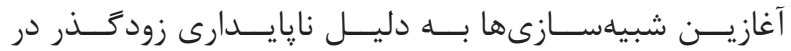

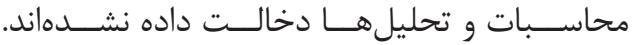

بافتهها

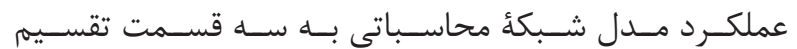

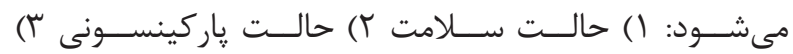

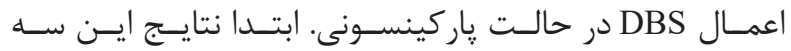

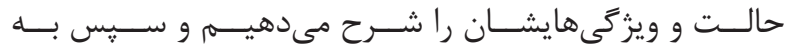

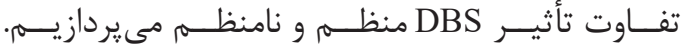

ويثزَى حالات سلامت، هار كينسون و DBS

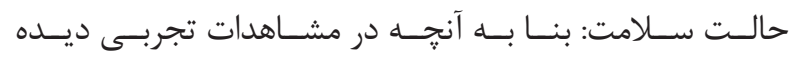

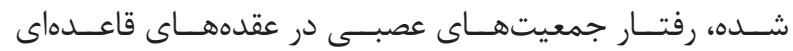

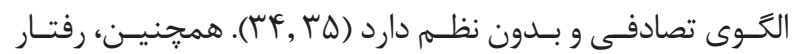

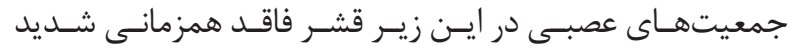

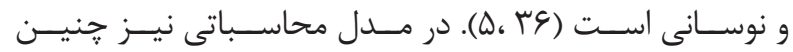

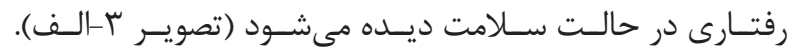

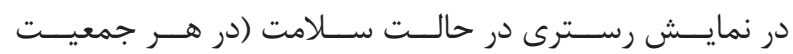

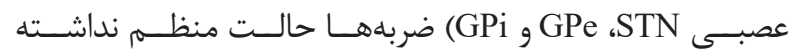

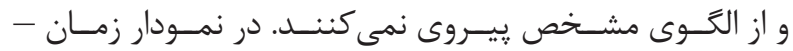

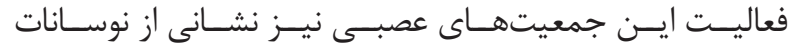

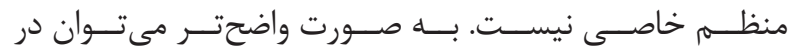

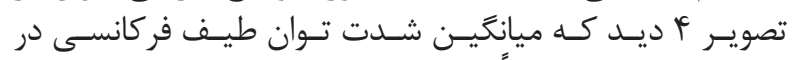

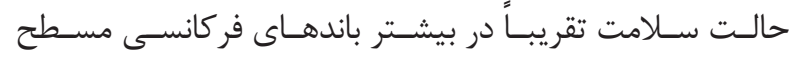

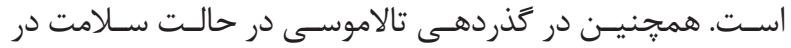

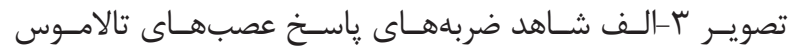

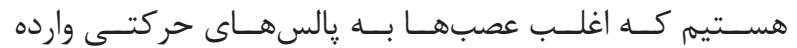

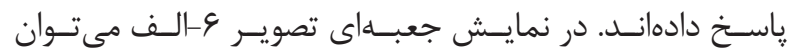

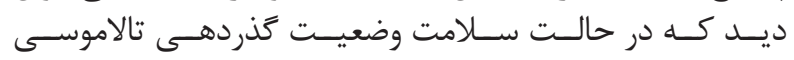

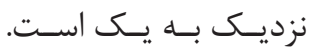

آزمون معنى دارى

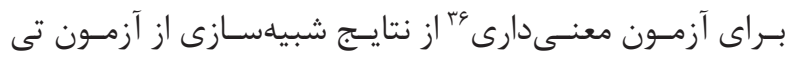

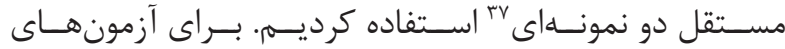

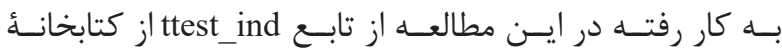
scipy

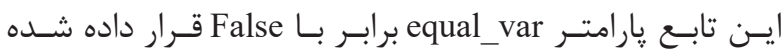

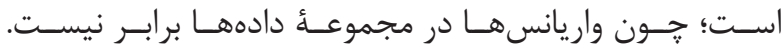

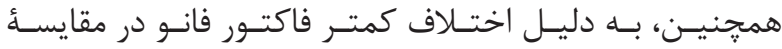

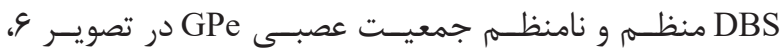

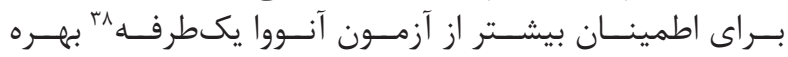

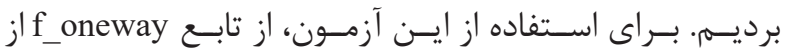

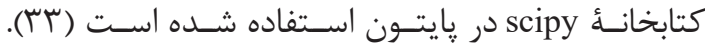

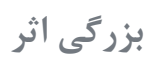

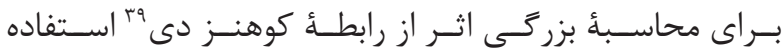

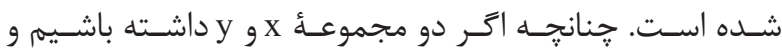

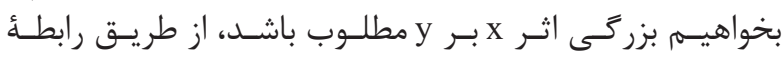

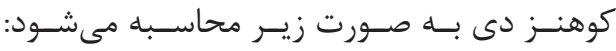

$$
\operatorname{Cohen}(x, y)=\frac{\operatorname{mean}(x)-\operatorname{mean}(y)}{\sqrt{\frac{\operatorname{std}(x)^{r}+\operatorname{std}(y)^{r}}{r}}}
$$

كــه در آن std، اسـتاندارد انحــرف معيــار و mean، ميانخيـنـ

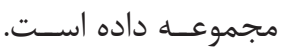

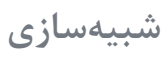

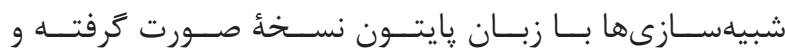

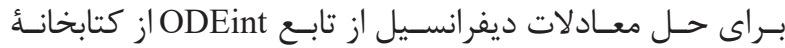
شئه Scipy

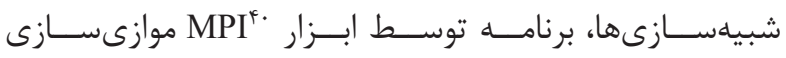

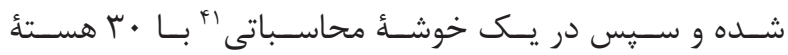

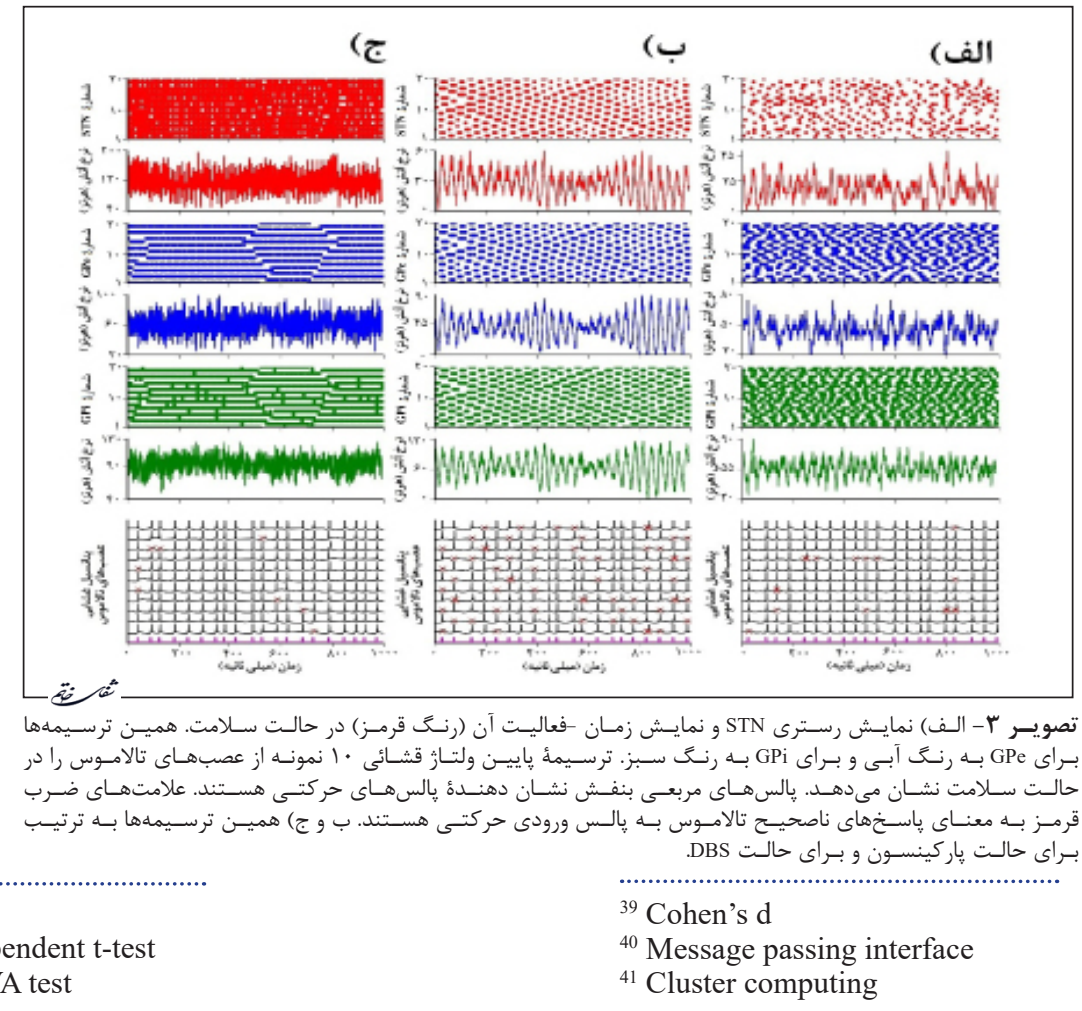

${ }^{36}$ Significance test

Two tailed independent t-test

One-way ANOVA test
${ }^{40}$ Message passing interface

${ }^{41}$ Cluster computing 


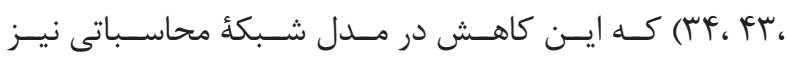

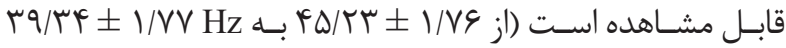

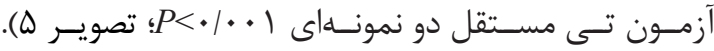

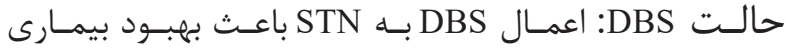

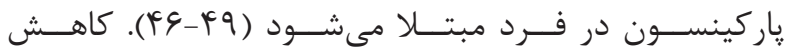

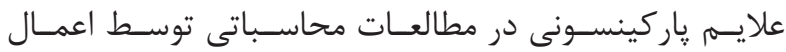
نشـان داده شــدهاند ( DBS

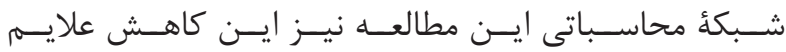

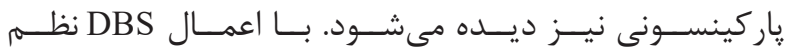

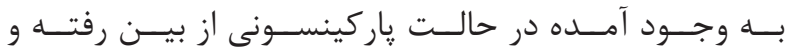

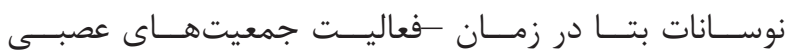

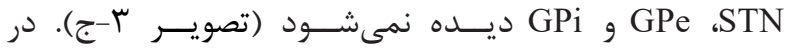

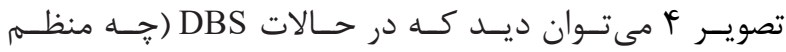

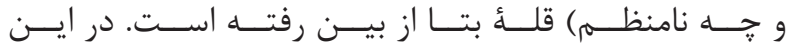

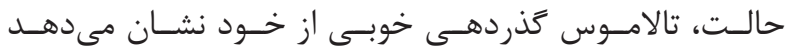

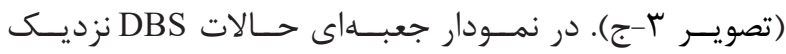

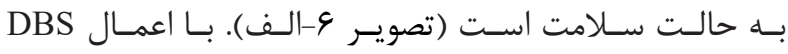

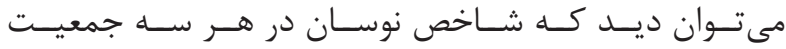

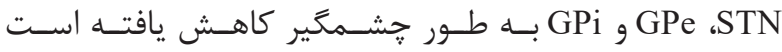

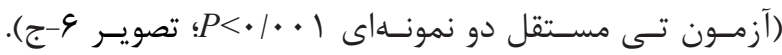

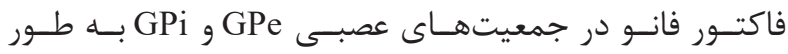

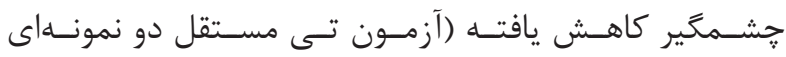

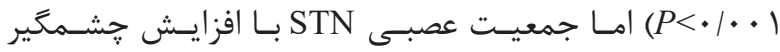

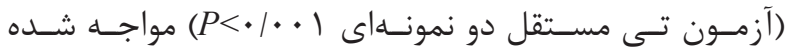

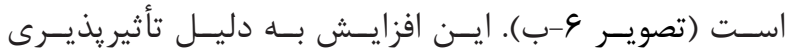

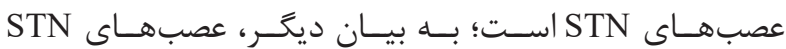

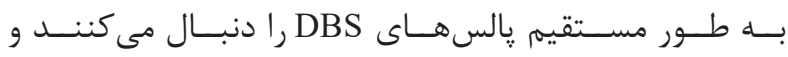

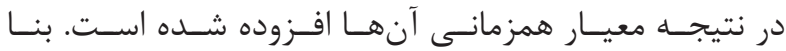

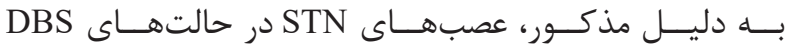

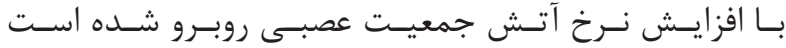

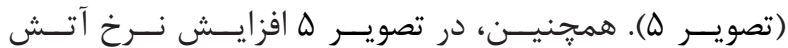

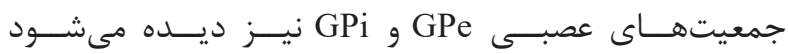

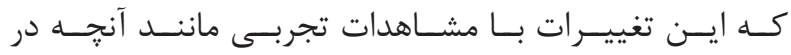

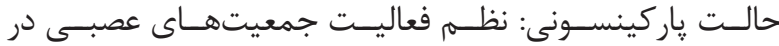

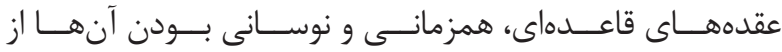

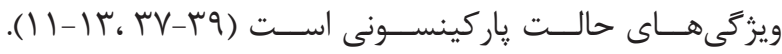

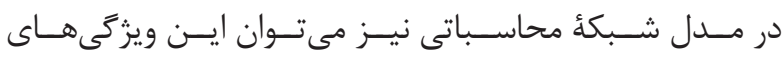

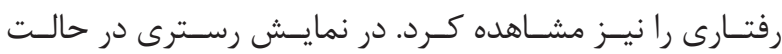

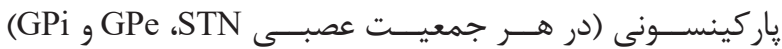

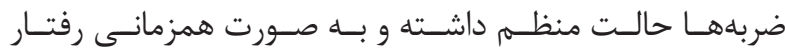

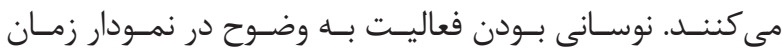

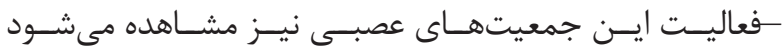

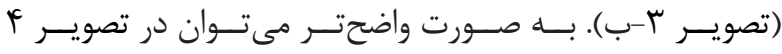

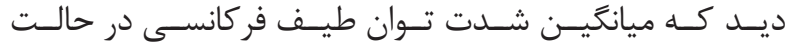

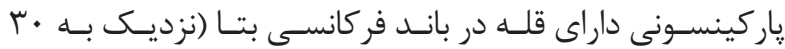

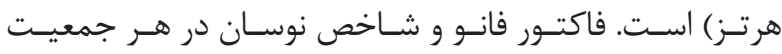

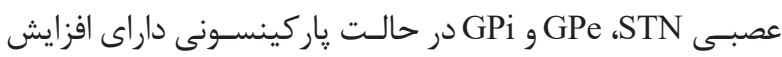

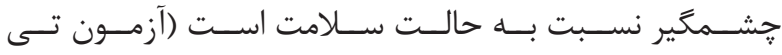

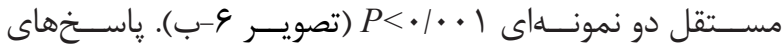

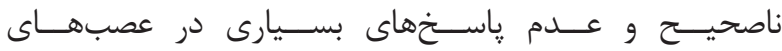

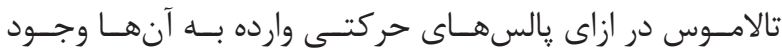

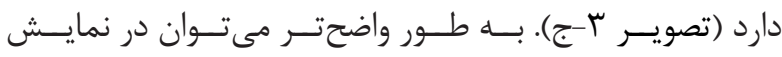

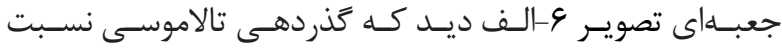

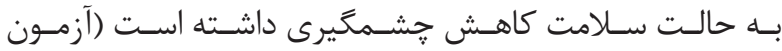

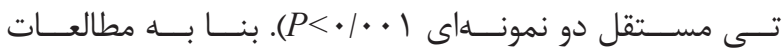

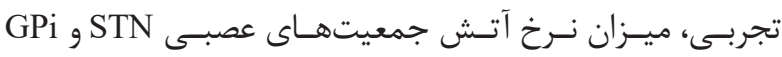

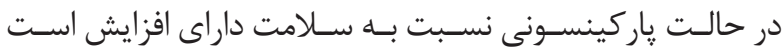

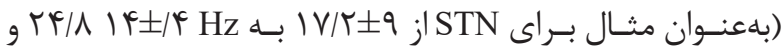

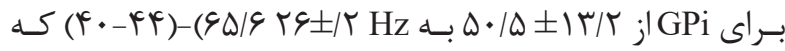

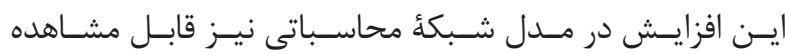

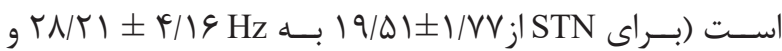
بـراى GPi از

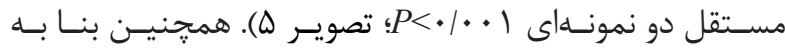

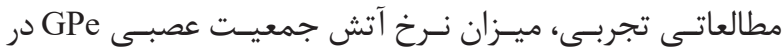

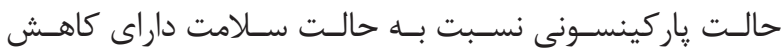

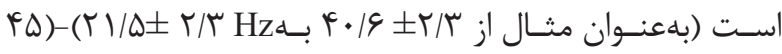

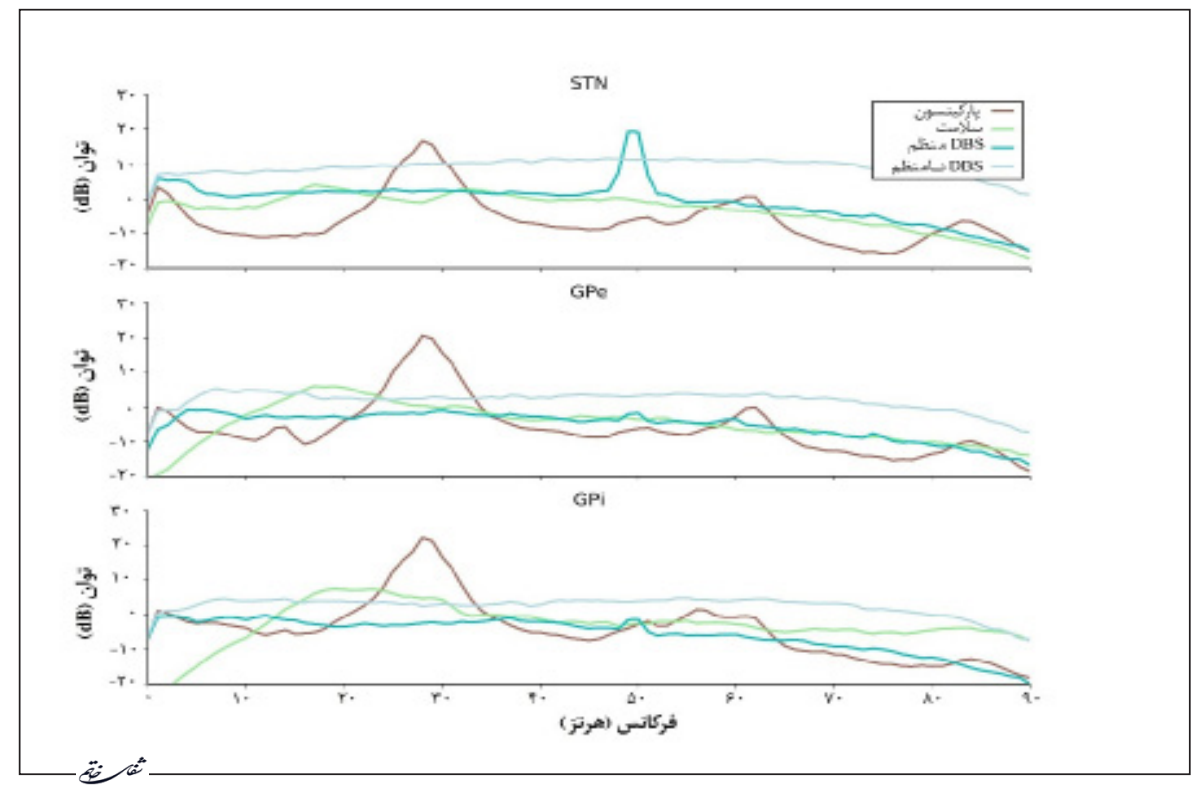




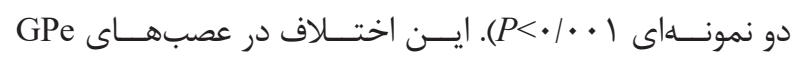

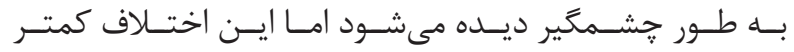

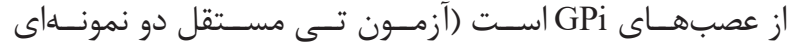

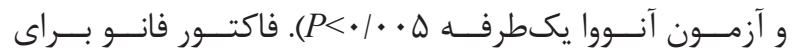

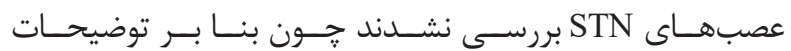

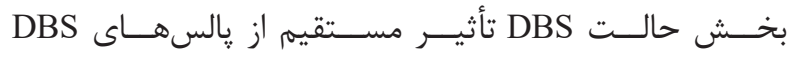

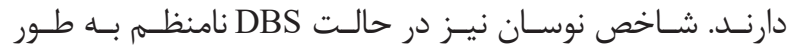

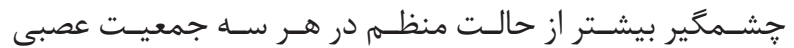

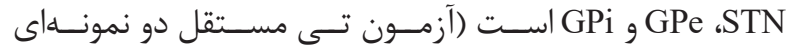

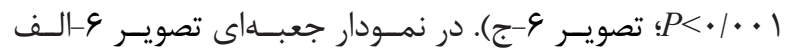

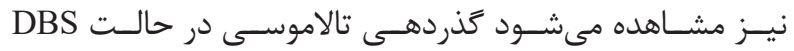

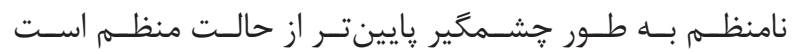

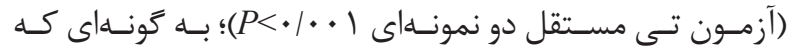

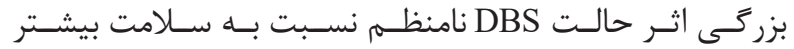

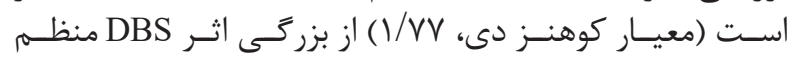

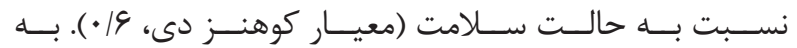

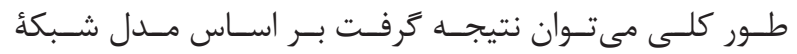

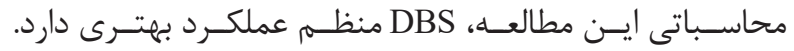

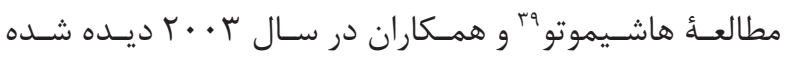

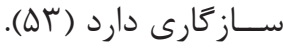

تفاوت DBS منظم و نامنظم

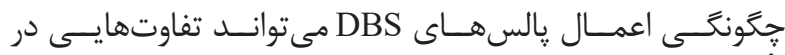

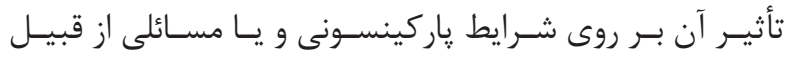

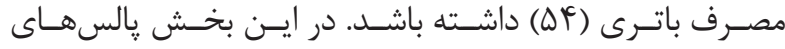

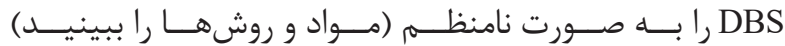

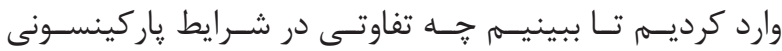

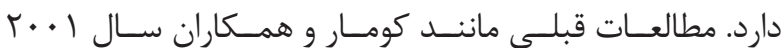

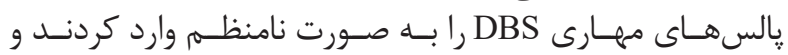

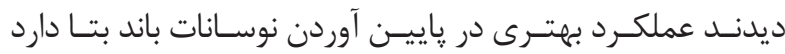

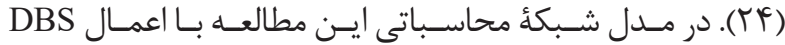

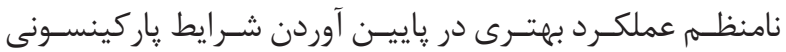

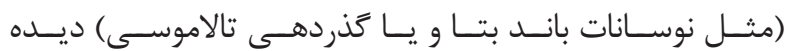

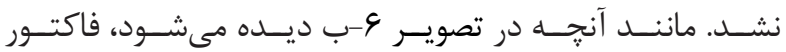

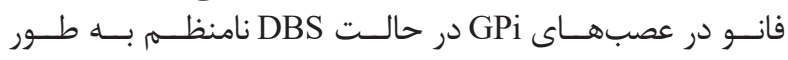

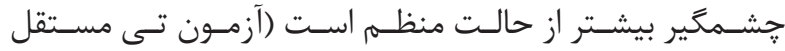

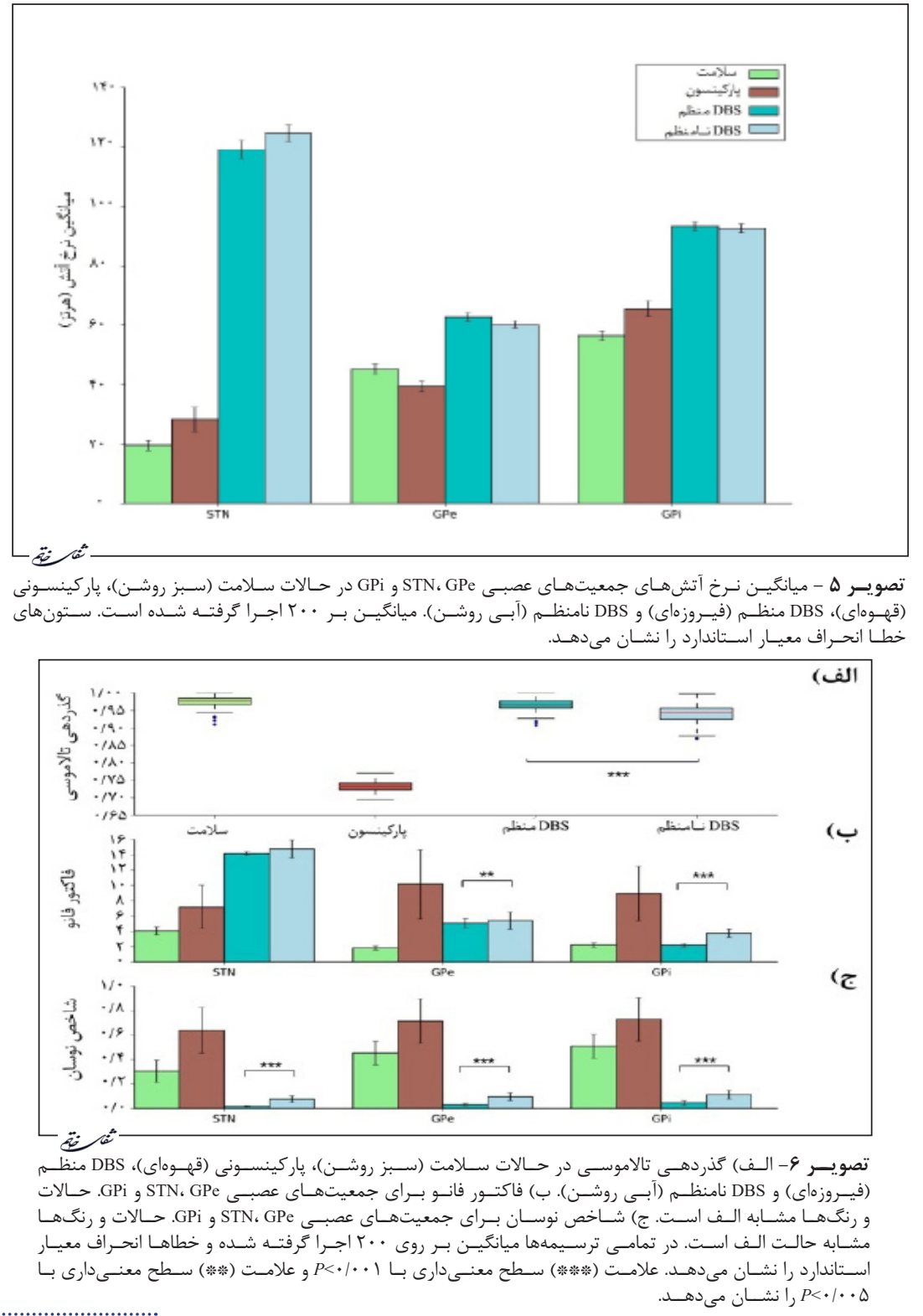

${ }^{39}$ Hashimoto 
عصبـى كلوبـوس ياليـدوس بــا آنجــهـ در هاشـيموتو و همــاران

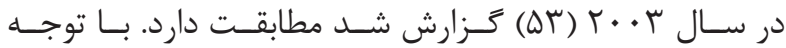

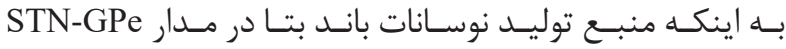

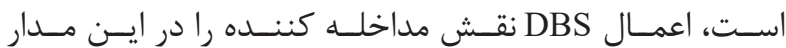

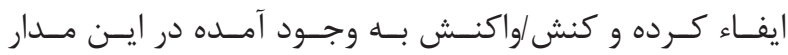

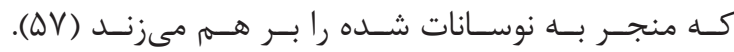
يالسهــاى DBS در مــدل شــبكة محاســباتى ايـن مطالعــهـ

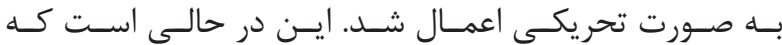

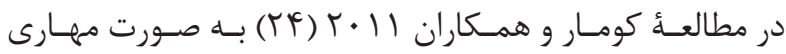

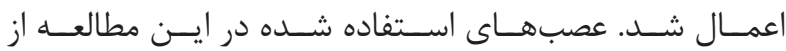

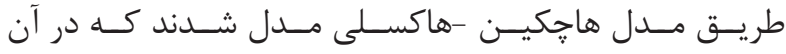

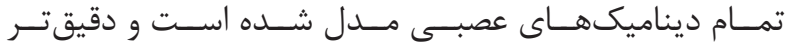

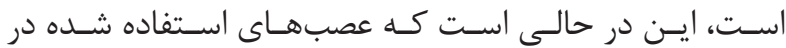

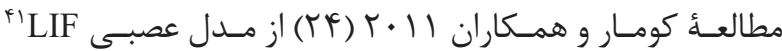

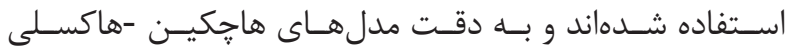

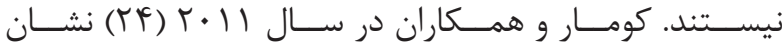

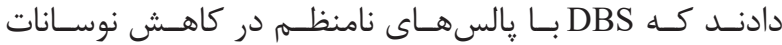

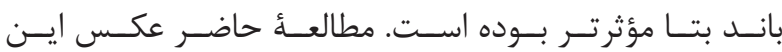

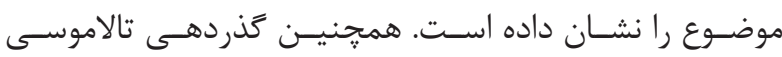

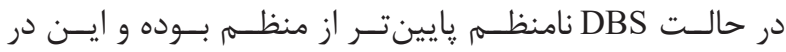

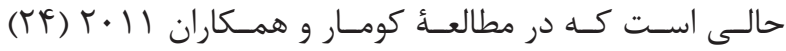

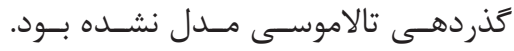

1. Squire LR, Berg D, Bhoom FE, Las SD, Ghosh A, Spitzer NC. Fundamental Neuroscience. $4^{\text {th }}$ ed. 2013; p. 1127.

2. Galvan A, Devergnas A, Wichmann T. Alterations in neuronal activity in basal ganglia-thalamocortical circuits in the parkinsonian state. Front Neuroanat. 2015; 9: 5

3. Liang L, DeLong MR, Papa SM. Inversion of dopamine responses in striatal medium spiny neurons and involuntary movements. J Neurosci. 2008; 28(30): 7537-47.

4. Mallet N, Ballion B, Le Moine C, Gonon F. Cortical inputs and GABA interneurons imbalance projection neurons in the striatum of parkinsonian rats. J Neurosci. 2006; 26: 3875-84

5. Miller WC, DeLong MR. Parkinsonian symptomatology. an anatomical and physiological analysis. Annals of the New York Academy of Sciences. $1988 ; \quad 515: 287-302$.

6. Baufreton J, Bevan MD. D2-like dopamine receptormediated modulation of activity-dependent plasticity at GABAergic synapses in the subthalamic nucleus. J Physiol. 2008; 586: 2121-42.

40 Tachibana

${ }^{41}$ Leaky integrated and fire 
103(5): 2707-16.

13. Wingeier B, Tcheng T, Koop MM, Hill BC, Heit $\mathrm{G}$, Bronte-Stewart HM. Intra-operative STN DBS attenuates the prominent beta rhythm in the STN in Parkinson's disease. Experimental Neurology. 2006; 197(1): 244-51.

14. Magill PJ, Bolam JP, Bevan MD. Dopamine regulates the impact of the cerebral cortex on the subthalamic nucleus-globus pallidus network. Neuroscience. 2001; 106(2): 313-30.

15. Plenz D, Kital ST. A basal ganglia pacemaker formed by the subthalamic nucleus and external globus pallidus. Nature. 1999; 400: 677-82.

16. Tachibana $\mathrm{Y}$, Iwamuro $\mathrm{H}$, Kita H, Takada $\mathrm{M}$, Nambu A. Subthalamo-pallidal interactions underlying parkinsonian neuronal oscillations in the primate basal ganglia. European Journal of Neuroscience. 2011; 34: $1470-84$

17. Benabid AL. Deep brain stimulation for Parkinson's disease. Current Opinion in Neurobiology. 2003; 13(6): 696-706.

18. Miyasaki JM. Practice parameter: evaluation and treatment of depression, psychosis, and dementia in Parkinson disease (an evidence-based review): report of the quality standards subcommittee of the american academy of neurology. Neurology. 2006; 66(7): 9961002.

19. Pahwa R, Factor Sa, Lyons KE, Ondo WG, Gronseth $\mathrm{G}$, Bronte-Stewart $\mathrm{H}$, et al. Practice parameter: treatment of parkinson disease with motor fluctuations and dyskinesia (an evidence-based review): report of the quality standards subcommittee of the american academy of neurology. Neurology. 2006; 66(7): 983-95.

20. Kringelbach ML, Jenkinson N, Owen SLF, Aziz TZ. Translational principles of deep brain stimulation. Nature reviews. Neuroscience. 2007; 8: 623-35.

21. McIntyre CC, Savasta M, Walter BL, Vitek JL. How does deep brain stimulation work? Present understanding and future questions. Journal of clinical neurophysiology : official publication of the American Electroencephalographic Society. Journal of Clinical neurophysiology. 2004; 21(1): 40-50.

22. Nambu A. Seven problems on the basal ganglia. Current Opinion in Neurobiology. 2008; 18(6): 595-604.

23. Terman D, Rubin JE. High frequency stimulation of the subthalamic nucleus eliminates pathological thalamic rhythmicity in a computational model. Journal of Computational Neuroscience. 2004; 16(3): 211-35.

24. Kumar A, Cardanobile S, Rotter S, Aertsen A. The role of inhibition in generating and controlling parkinson's disease oscillations in the basal ganglia. Front Syst Neurosci. 2011; 5: 86 doi.org/10.3389/ fnsys.2011.00086.

25. So RQ, Kent AR, Grill WM. Relative contributions of local cell and passing fiber activation and silencing to changes in thalamic fidelity during deep brain stimulation and lesioning: a computational modeling study. Journal of Computational Neuroscience. 2012; 32: 499-519. doi. org/10.3389/fnsys.2011.00086.

26. Hahn PJ, Mcintyre CC. Modeling shifts in the rate and pattern of subthalamopallidal network activity during deep brain stimulation. Journal of Computational Neuroscience. 2011; 28(3): 425-41.

27. Izhikevich EM. Dynamical systems in neuroscience: the geometry of excitability and burtsing. Cambridge, Massachusetts London: England. 2007: p. 441.

28. Izhikevich EM. Which model to use for cortical spiking neurons? IEEE Transactions on Neural Networks. 2004; 15(5): 1063-70.

29. Terman D, Rubin JE, Yew aC, Wilson CJ. Activity patterns in a model for the subthalamopallidal network of the basal ganglia. J Neurosci. 2002; 22(7): 2963-76.

30. Park C, Worth RM, Rubchinsky LL. Neural dynamics in Parkinsonian brain: the boundary between synchronized and nonsynchronized dynamics. Physical Review E. 2011; 83: 1-4.

31. Ahn S, Zauber SE, Worth RM, Rubchinsky LL. Synchronized beta-band oscillations in a model of the globus pallidus-subthalamic nucleus network under external input. Front Comput Neurosci. 2016; 10: 1-12.

32. Kumar A, Schrader S, Aertsen A, Rotter S. The high-conductance state of cortical Networks. Neural Computation. 2008; 20(1): 1-43.

33. Eric Jones, Travis Oliphant, Pearu Peterson. SciPy: open source scientific tools for Python. 2001; http:// www.scipy.org/.

34. Pan HS, Walters JR. Unilateral lesion of the nigrostriatal pathway decreases the firing rate and alters the firing pattern of globus pallidus neurons in the rat. Synapse. 1988; 2: 650-6.

35. Raz A, Vaadia E, Bergman H. Firing patterns and 
correlations of spontaneous discharge of pallidal neurons in the normal and the tremulous 1-methyl4-phenyl-1,2,3,6-tetrahydropyridine vervet model of parkinsonism. J Neuroscience. 2000; 20(22): 8559-71

36. Brown P, Kupsch A, Magill PJ, Sharott A, Harnack D, Meissner W. Oscillatory local field potentials recorded from the subthalamic nucleus of the alert rat. Experimental Neurology. 2002; 177(2): 581-5.

37. Beck MH, Haumesser JK, Kuhn J, Altschuler J, Kuhn AA, van Riesen C. Short- and long-term dopamine depletion causes enhanced beta oscillations in the cortico-basal ganglia loop of parkinsonian rats. Experimental Neurology. 2016; 286: 124-36.

38. Brown P, Williams D. Basal ganglia local field potential activity: character and functional significance in the human. Clinical Neurophysiology. 2005; 116(11): 2510-9.

39. Wilson CL, Cash D, Galley K, Chapman H, Lacey MG, Stanford IM. Subthalamic nucleus neurones in slices from 1-methyl-4-phenyl-1,2,3,6-tetrahydropyridinelesioned mice show irregular, dopamine-reversible firing pattern changes, but without synchronous activity. Neuroscience. 2006; 143(2): 565-72.

40. Bergman H, Wichmann T, Karmon B, DeLong MR. The primate subthalamic nucleus. II. neuronal activity in the MPTP model of parkinsonism. Journal of Neurophysiology. 1994; 72(2): 507-20.

41. Hassani OK, Mouroux M, Féger J. Increased subthalamic neuronal activity after nigral dopaminergic lesion independent of disinhibition via the globus pallidus. Neuroscience. 1996; 72(1): 105-15.

42. Hutchison WD, Lozano AM, Davis KD, Saint-Cyr JA, Lang AE, Dostrovsky JO. Differential neuronal activity in segments of globus pallidus in Parkinson's disease patients. Neuroreport. 1994; 5(12): 1533-7.

43. Miller WC, DeLong MR. Altered tonic activity of neurons in the globus pallidus and subthalamic nucleus in the primate MPTP Model of parkinsonism. Carpenter MB, Jayaraman A. The basal ganglia ii: structure and function-current concepts. Boston, MA: Springer US; 1987. p. 415-27.

44. Wichmann T, Bergman H, Starr PA, Subramanian T, Watts RL, DeLong MR. Comparison of MPTP-induced changes in spontaneous neuronal discharge in the internal pallidal segment and in the substantia nigra pars reticulata in primates. Experimental Brain Research. 1999; 125(4): 397-409.
45. Sterio D, Beric A, Dogali M, Fazzini E, Alfaro G, Devinsky O. Neurophysiological properties of pallidal neurons in palanson 's disease. Annals of Neurology. 1994; 35(5): 586-91.

46. Giannicola G, Marceglia S, Rossi L, Mrakic-Sposta S, Rampini P, Tamma F, et al. The effects of levodopa and ongoing deep brain stimulation on subthalamic beta oscillations in Parkinson's disease. Experimental Neurology. 2010; 226(1): 120-7.

47. Groiss SJ, Wojtecki L, Südmeyer M, Schnitzler A. Deep brain stimulation in Parkinson's disease. Therapeutic Advances in Neurological Disorders. 2009; 2: $20-8$.

48. Weaver FM, Follett KA, Stern M, Hur K, Harris CL, Marks Jr, et al. Bilateral deep brain stimulation vs best medical therapy for patients with advanced parkinson disease. The Journal of the American Medical Association. 2009; 301: 301(1): 63-73.

49. Wichmann T, DeLong MR. Deep brain stimulation for neurologic and neuropsychiatric disorders. Neuron. 2006; 52(1): 197-204.

50. Agarwal R, Sarma SV. The effects of DBS patterns on basal ganglia activity and thalamic relay: a computational study. Journal of Computational Neuroscience. 2012; 33(1): 151-67.

51. Guo Y, Rubin JE, McIntyre CC, Vitek JL, Terman D. Thalamocortical relay fidelity varies across subthalamic nucleus deep brain stimulation protocols in a data-driven computational model. Journal of Neurophysiology. 2008; 99(3): 1477-92.

52. Schiff SJ. Towards model-based control of Parkinson's disease. Philosophical Transactions of the Royal Society A: Mathematical, Physical and Engineering Sciences. 2010; 368(1918): 2269-308.

53. Hashimoto T, Elder CM, Okun MS, Patrick SK, Vitek JL. Stimulation of the subthalamic nucleus changes the firing pattern of pallidal neurons. J Neurosci. 2003; 23(5): 1916-23.

54. Anheim M, Fraix V, Chabardès S, Krack P, Benabid AL, Pollak P. Lifetime of itrel II pulse generators for subthalamic nucleus stimulation in Parkinson's disease. Movement Disorders. 2007; 22(16): 2436-9.

55. Best J, Park C, Terman D, Wilson C. Transitions between irregular and rhythmic firing patterns in excitatory-inhibitory neuronal networks. Journal of Computational Neuroscience. 2007; 23(2): 217-35. 


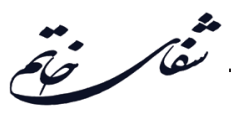

56. Feng X-j, Greenwald B, Rabitz H, Shea-Brown E, Kosut R. Toward closed-loop optimization of deep brain stimulation for Parkinson's disease: concepts and lessons from a computational model. Journal of Neural Engineering. 2007; 4(2): L14.
57. The Deep-Brain Stimulation for Parkinson's Disease Study Group. Deep-brain stimulation of the subthalamic nucleus or the pars interna of the globus pallidus in Parkinson's disease. New England Journal of Medicine. 2001; 345: 956-63. 